\title{
First detection of the FSRQ TON 0599 in the VHE regime
}

\author{
Joe Hirako* \\ Kyoto University \\ E-mail: hirako.joe.53cest.kyoto-u.ac.jp \\ Tomislav Terzic \\ University of Rijeka \\ E-mail: tomislav.terzicegmail.com
}

\author{
Antonio Stamerra \\ INAF \\ E-mail: antonio.stamerra@inaf.it
}

\section{Fabrizio Tavecchio}

INAF

E-mail: fabrizio.tavecchio@bera.inaf.it

\section{Chiara Righi}

INAF

E-mail: chiara.righi@inaf.it

\section{Claudia M. Raiteri}

INAF

E-mail: claudia.raiterieinaf.it

\section{Luigi Pacciani}

INAF

E-mail: luigi.pacciani@inaf.it

TON 0599 is the seventh flat spectrum radio quasar (FSRQ) detected so far in the very high energy (VHE, E $>100 \mathrm{GeV}$ ) regime. The VHE gamma-ray emission was discovered on December 2017 with the MAGIC telescopes, making TON 0599 the third farthest source $(z=0.72)$ in the VHE sky. The MAGIC observation was triggered by a hardening of the high energy (HE, E > $100 \mathrm{MeV}$ ) gamma-ray spectrum observed with Fermi-LAT and during one hour, the flux reached 40 per cent of the Crab Nebula flux above $80 \mathrm{GeV}$. Observations continued for two weeks, with a gradual decrease of the flux. The simultaneous VHE (MAGIC) and HE (Fermi) gamma-ray spectra connect smoothly. The joint spectrum shows a sudden drop above $\sim 8 \mathrm{GeV}$, which may suggest a strong absorption of the VHE gamma rays within the source, and intra-day spectral variations. MAGIC data provide a unique opportunity to investigate these variations during a flaring episode and peek into the structure of FSRQs. We round the multiwavelength picture with observations in optical, IR, and radio bands acquired by the WEBT collaboration during the whole 2017-2018 optical observing season, from November to May. 
International Conference on Black Holes as Cosmic Batteries: UHECRs and Multimessenger Astronomy BHCB2018

12-15 September, 2018

Foz du Iguazu, Brasil

${ }^{*}$ Speaker. 


\section{INTRODUCTION}

Active galactic nuclei (AGNs) are usually described as accreting supermassive black holes in the center of galaxies, as proposed in the so-called unification model [1]. In some cases, two jets of ultra-relativistic particles are emitted in the vicinity of the center. Jetted-AGNs with a jet almost aligned to Earth are identified as blazars. Blazars are known for their energetic activity observed within a wide wavelength range from radio to very high energy (VHE, E $>100 \mathrm{GeV}$ ) gamma rays. They are classified into two classes: flat spectrum radio quasar (FSRQ) and BL Lac (BL Lacertae object). FSRQs have strong optical emission lines with broad equivalent widths in contrast to BL Lacs. Both types of blazars follow a similar trend in their spectral energy distributions (SEDs), which show two bumps. The spectra are thought to represent emission from high energy charged particles. In the leptonic model, the lower peak is described by synchrotron radiation of electrons, the higher one is due to synchrotron self-Compton (SSC) process. Additionally, in the case of FSRQs, external Compton (EC) components are inferred to be needed for modeling the SEDs. EC is a process where external field photons are up-scattered by the accelerated electrons. There are several candidate sources of external seed photons e.g. a dust torus, a broad line region (BLR), or an accretion disk. An additional issue is where the emission region is located. Possible candidates are: within the BLR, in the radiation field of the dust torus, and near the radio blob. If it is closer to SMBH, it is easier to explain the FSRQ's flux variability and luminosity, but the absorption of sub-TeV gamma rays is more problematic (eg. [2], [3]). One of the approaches to constrain the emission region is to calculate the timescales of the flux changes. We can calculate the timescale $t_{v a r}$ as in [4], and then evaluate the radius of the emission region as $R_{e m i t} \leq c \delta t_{v a r} /(1+z)$, where $c$ is the speed of light, $\delta$ is the Doppler factor of the emission region, and $z$ is the redshift.

On 2017 December 15, the FSRQ TON 0599 was observed for the first time in the VHE regime with the MAGIC telescope, triggered by a hardening the spectral index in high energy (HE, E > $100 \mathrm{MeV}$ ) seen with Fermi-LAT. In this contribution, we will show the results of the observations at VHE gamma rays with the MAGIC telescopes, combined with data in HE with Fermi-LAT, X-ray band with Swift-XRT, and optical, IR, and radio bands by the WEBT collaboration. We will also present the result of our modeling applied to the multiwavelength data.

\section{THE FSRQ TON 0599}

TON 0599 is located at a right ascension of $11 \mathrm{~h} 59 \mathrm{~m} 31.8 \mathrm{~s}$ and declination of $+29 \mathrm{~d} 14 \mathrm{~m}$ 45s [5], with redshift $z=0.724$ [6], which makes it the third farthest source in the VHE sky. The apparent jet opening angle is $2.0^{\circ}$ [7], and the disk luminosity is $3.6 \times 10^{45} \mathrm{erg} / \mathrm{s}$ [8].

From 1966 to 2010, a long-term observations program had been conducted at the University of Michigan Radio Astronomy Observatory (UMRAO) in radio bands of 4.8, 8.0, and 14.5 $\mathrm{GHz}$ [9], and long-term flux variations were observed. In [10], three numerical analyses (the LS periodogram method, the Weighted Wavelet Z-transform method, and the Phase Dispersion Minimization method) were employed for the long-term radio light curves, and found four harmonic oscillating components with periods of 1.7, 2.3, 3.4 and 7.7 years respectively. These periodicities are possibly related to the propagation of the dynamics of accretion disk, but this is still unclear. 


\section{OBSERVATIONS WITH MAGIC}

MAGIC is a stereo system of two Imaging Atmospheric Cherenkov Telescopes (IACTs) with two $17 \mathrm{~m}$ mirror dishes, located in the Canary Island of La Palma $\left(28.7^{\circ} \mathrm{N}, 17.9^{\circ} \mathrm{W}\right)$, at the height of $2200 \mathrm{~m}$ a.s.l. [11]. After the first detection of TON 0599 on 2017 December 15 (MJD 58102), MAGIC continued to observe the source until 2017 December 29 (MJD 58116). We selected a total of $19.2 \mathrm{~h}$ of good quality data according to the weather conditions and used MARS, the standard data analysis package of MAGIC for the data analysis [12, 13]. A clear signal with a statistical significance of 10.4 sigma was detected during $0.96 \mathrm{~h}$ of exposure already in the first night of observation (Fig. 1, left).
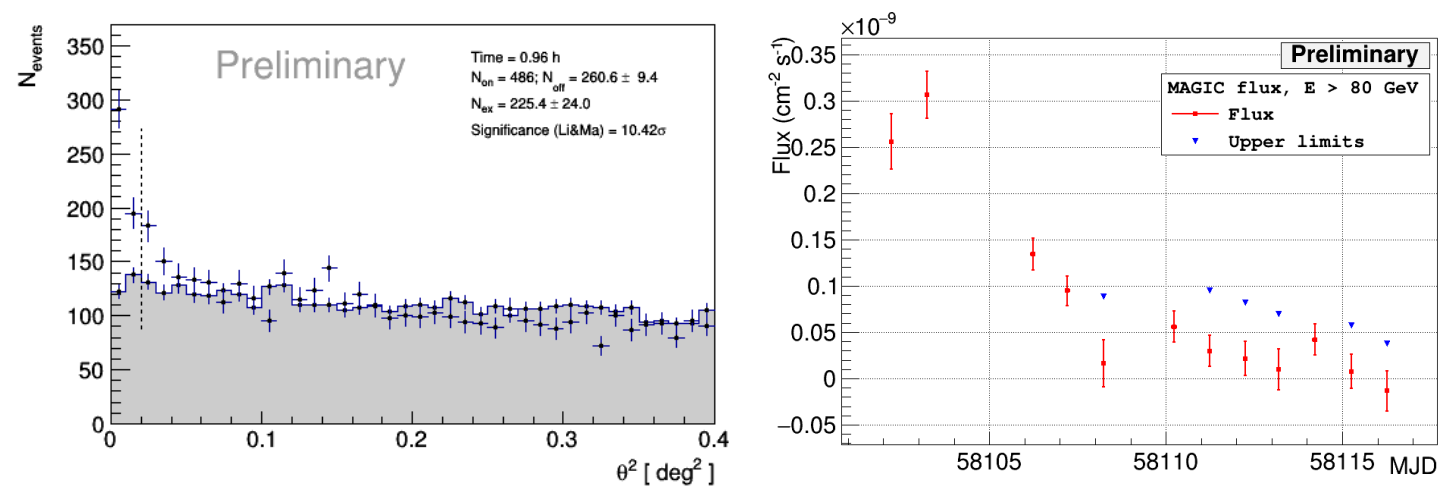

Figure 1: Left : MAGIC observations on MJD 58102. $N_{\text {on }}$ is the number of all events with $\theta^{2}<0.02 \mathrm{deg}^{2}$ (vertical dashed line) with respect to the source position in the camera, and $N_{\text {off }}$ the normalized number of all events from the same region around OFF-source position.

Right : Integral flux measured by MAGIC above $80 \mathrm{GeV}$ for the whole set of observations. Blue triangles represent $95 \%$ confidence level upper limits for points whose significance is less than $2 \sigma$.

\section{DATA ANALYSIS AND RESULTS}

\subsection{MAGIC and Fermi-LAT results}

MAGIC light curve above $80 \mathrm{GeV}$ for the whole period is shown in Fig. 1, right. The flux reached its maximum on the second night of observation, then gradually decreased until the last observation. For the sake of investigating the spectral variation along with the flare fading, we divided the data into four sub-samples named as periods A to D based on the flux level. Periods A and B correspond to the first two nights (MJD 58102 and 58103) respectively when the flux was at its highest levels. Period C contains MJD 58106 and 58107 (3rd and 4th point in the MAGIC light curve), which correspond to intermediate flux levels. Period D contains all the other nights: from MJD 58108 to 58116, and is the period of lowest flux levels. We calculated the spectra for each of the four periods separately, combining MAGIC data with simultaneous data from FermiLAT. The corresponding SEDs are displayed in Fig. 2. The MAGIC data points in Fig. 2 represent the intrinsic spectra. The gamma-ray absorption by the extragalactic background light (EBL), was corrected adopting the model [14]. For all four periods, we see a smooth connection between the 

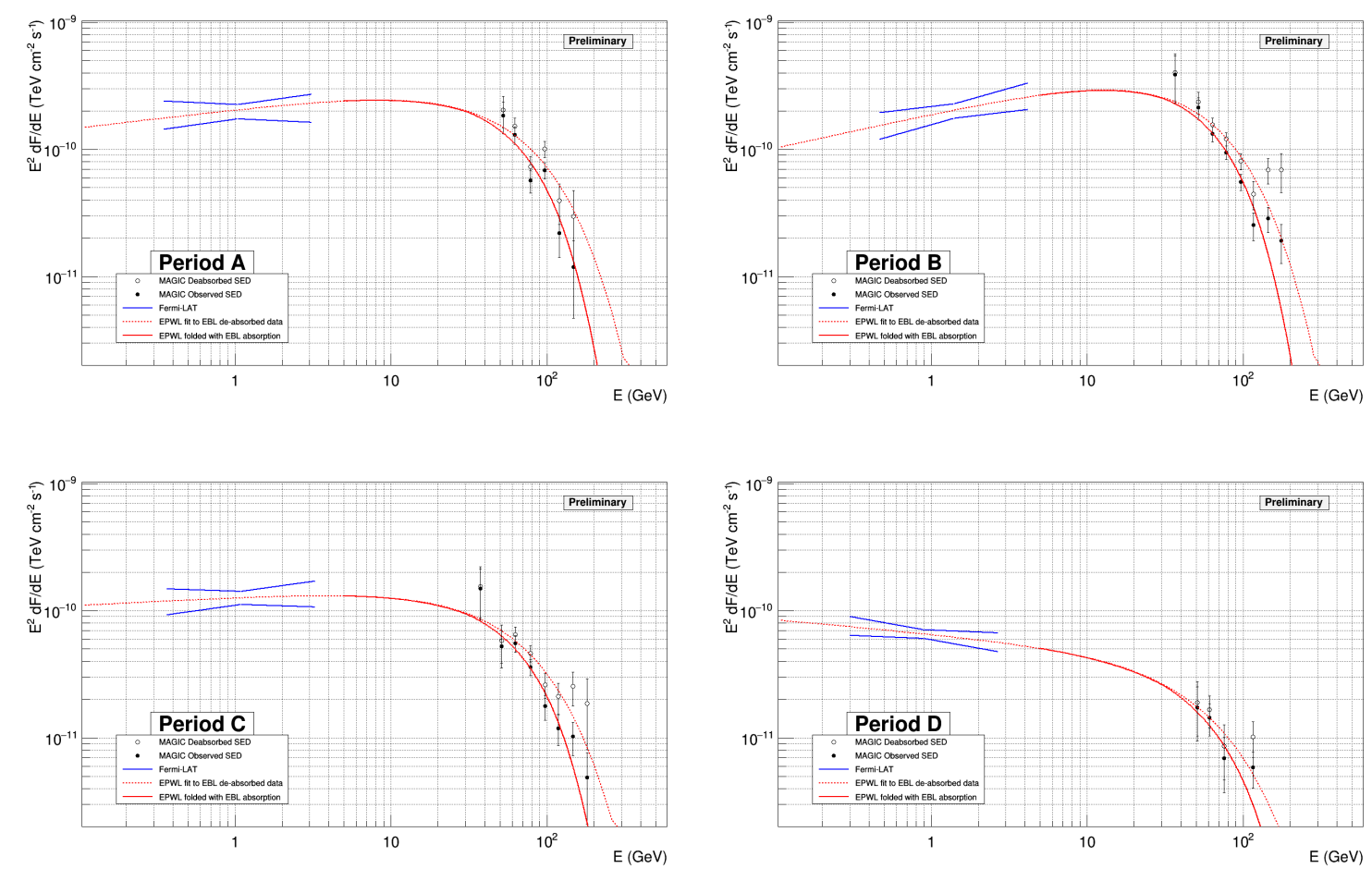

Figure 2: Spectral energy distributions for Periods A through D. Blue lines are Fermi-LAT spectra with errors. Solid black circles are MAGIC observed points, and hollow black circles are de-absorbed points. Red lines are fitting curves for MAGIC observed points, and dotted red lines are for de-absorbed points. The fitting function is a power-law with an exponential cut off.

Fermi-LAT to the MAGIC energy bands. Those figures also indicate that the photon index had been changing as the period shifts.

\subsection{Multiwavelength results}

\subsubsection{Multiwavelength light curves}

The multiwavelength light curve and the variation of polarization in the optical band are shown in Fig. 3, left. We can see the similar flux variations in the VHE, HE, and optical bands, while the flux in the radio band was almost stable around the flare. The interesting point, unique to this source, is that the polarization percentage increased after the flare.

\subsubsection{Multiwavelength SED}

We gathered the multiwavelength data points of the SEDs and adopted a simple leptonic model to describe each SED (Fig. 3, right). The parameters we used are listed in Table 1. We assumed a single emission region outside of BLR and an EC component explained by an extra photon field from a dust torus modeled by a black body radiation at $1000 \mathrm{~K}$. In order to allow emission above $20 \mathrm{GeV}$, we assumed that the emission region is beyond the BLR, so we fixed the distance $d$ between emitting region from the $\mathrm{SMBH}$, as $d=10 \times R_{\text {emit }}$ in the calculation. That is consistent with a size of BLR $R_{B L R}=1.9 \times 10^{17} \mathrm{~cm}$ and a size of dust torus $R_{\text {torus }}=4.7 \times 10^{18} \mathrm{~cm}$, calculated with the 
method of [15]. We used a broken power-law function for modeling the intrinsic energy distribution of the electrons:

$$
f(\gamma)= \begin{cases}\gamma^{-n_{1}} & \left(\gamma<\gamma_{\text {break }}\right) \\ \gamma^{-n_{2}} & \left(\gamma>\gamma_{\text {break }}\right)\end{cases}
$$

and,

$$
N=\int_{\gamma_{\min }}^{\gamma_{\max }} n(\gamma) d \gamma=\int_{\gamma_{\min }}^{\gamma_{\max }} K f(\gamma) d \gamma
$$

Where $N$ is a number of electrons per unit volume and $K$ is a normalization factor. In the modeling, synchrotron emission accounts for the low-energy bump, up to the "valley" between the two humps, and SSC emission contributes substantially (about 50\%) only in the X-ray band. Above the X-ray band, the emission is dominated by EC on the torus photons. From Table 1, we got the result that the jet was slowing down along the flare, as $\Gamma$ become smaller with time.
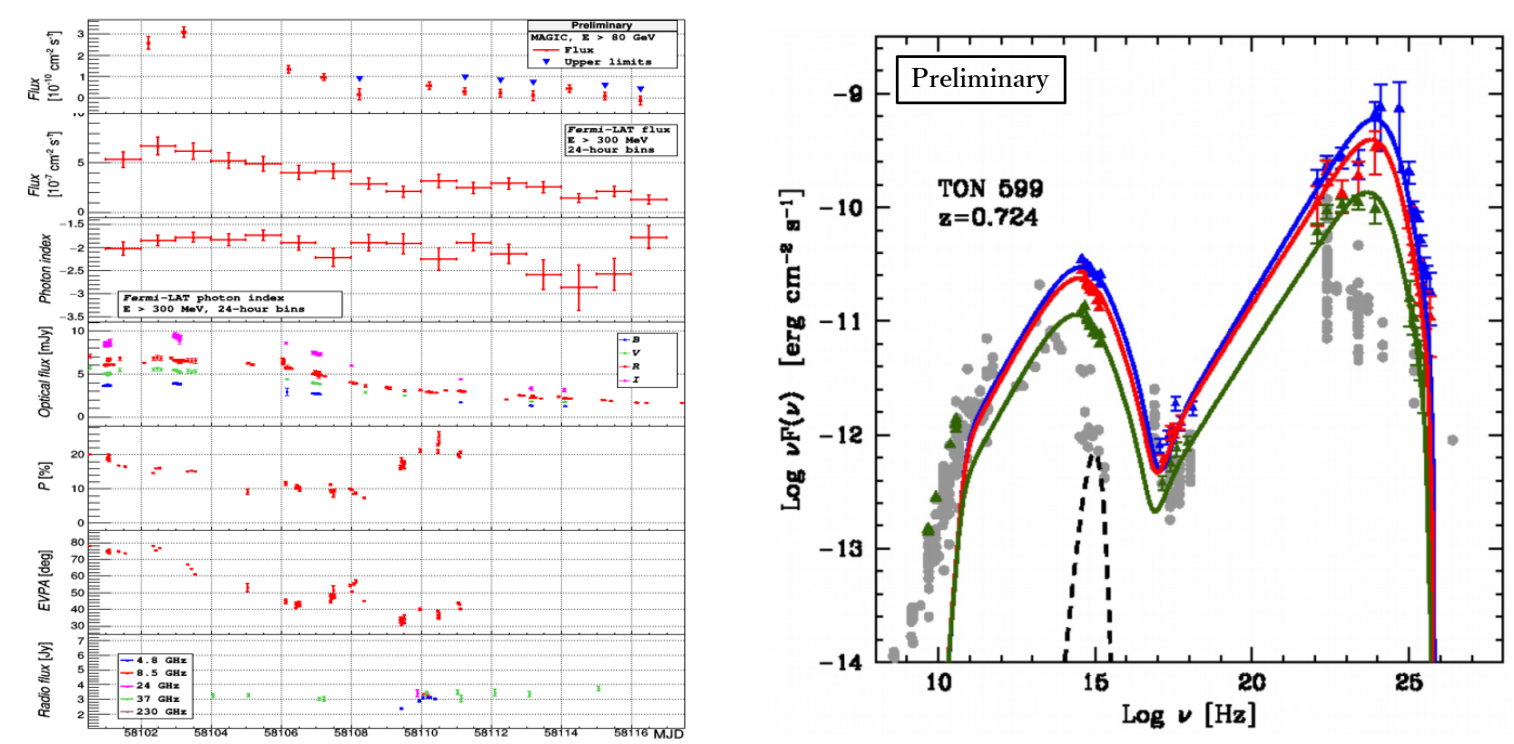

Figure 3: Left : Preliminary multiwavelength light curves. In order from the top, VHE flux (MAGIC), HE flux and photon index (Fermi-LAT), optical flux (WEBT), optical polarization (WEBT), electric vector position angle (WEBT), and radio flux (WEBT).

Right : Preliminary multiwavelength SED with models. VHE, HE, X-ray, optical and radio data are included. Each color corresponds to a different period. Blue: the sum of periods A and B, red: period C, green: period D, gray: historical data, and black dotted line: radiation from the dust torus at $1000 \mathrm{~K}$.

\section{CONCLUSION}

The MAGIC telescopes detected FSRQ TON599, the third farthest source in VHE sky at $z=0.724$ for the first time on 15th December 2017 during a flaring state. MAGIC continued observations until 29th December and we could see large flux variations as shown in Fig. 1, right. 


\begin{tabular}{cccc}
\hline & \multicolumn{3}{c}{ Period } \\
Parameter & A \& B & C & D \\
\hline$\gamma_{\text {min }}$ & 3.0 & 3.0 & 3.0 \\
$\gamma_{\text {break }}$ & $7.5 \times 10^{3}$ & $7.5 \times 10^{3}$ & $7.5 \times 10^{3}$ \\
$\gamma_{\text {max }}$ & $5.0 \times 10^{4}$ & $5.0 \times 10^{4}$ & $5.0 \times 10^{4}$ \\
$n_{1}$ & 2.0 & 2.0 & 2.0 \\
$n_{2}$ & 4.8 & 4.8 & 4.8 \\
$B[\mathrm{G}]$ & 0.20 & 0.18 & 0.18 \\
$K\left[\mathrm{~cm}^{-3}\right]$ & $3.0 \times 10^{3}$ & $3.9 \times 10^{3}$ & $3.7 \times 10^{3}$ \\
$R_{\text {emit }}[\mathrm{cm}]$ & $5.8 \times 10^{16}$ & $6.0 \times 10^{16}$ & $6.0 \times 10^{16}$ \\
$d[\mathrm{~cm}]$ & $5.8 \times 10^{17}$ & $6.0 \times 10^{17}$ & $6.0 \times 10^{17}$ \\
$\delta$ & 23.0 & 20.9 & 17.6 \\
$\Gamma$ & 15 & 12 & 10
\end{tabular}

Table 1: Parameters used to models the SED of TON 0599 for each period. We used a disk luminosity $L_{\text {disk }}=3.6 \times 10^{45}[\mathrm{erg} / \mathrm{s}]$ from [8]. B : magnetic field along with the jet, $\delta$ : bulk Doppler factor, $\Gamma$ : bulk Lorenz factor.

We divided MAGIC data in periods according to the flux level as explained in section 4.1. For each period, we calculated the high energy SED with corresponding Fermi-LAT data and we found the variation of the spectrum depicted in Fig. 2. In the HE band, a power-law with an exponential cut off function is favored to fit the spectral shape. From the comparison with the other bands, we found that the flux follows the same trend in VHE, HE and optical, and the polarization percentage changed after the flare (Fig. 3, left). The SEDs from three different periods were modeled with a simple leptonic model (Fig. 3, right and Table 1). The data are consistent with a jet slowing down along with the flaring episode. As a results, the we got a nice consistence with a simple leptonic model and concluded that the jet was slowing down along with the flare. We will continue to investigate those interesting results and discuss the mechanisms at work in our future work.

\section{References}

[1] C. M. Urry and P. Padvani, Unified schemes for radio-loud active galactic nuclei, Astronomical Society of the Pacific 107 (1995) 803.

[2] G. Ghisellini, F. Tavecchio, G. Bodo and A. Celotti, TeV variability in blazars: how fast can it be?, Monthly Notices of the Royal Astronomical Society: Letters 393 (2009) L16.

[3] M. Sikora, Łukasz Stawarz, R. Moderski, K. Nalewajko and G. M. Madejski, Constraining emission models of luminous blazar sources, The Astrophysical Journal 704 (2009) 38.

[4] Y. H. Zhang, A. Celotti, A. Treves, L. Chiappetti, G. Ghisellini, L. Maraschi et al., Rapid X-ray variability of the BL Lacertae object PKS 2155-304, The Astrophysical Journal 527 (1999) 719.

[5] K. J. Johnston, A. L. Fey, N. Zacharias, J. L. Russell, C. Ma, C. d. Vegt et al., A radio reference frame, The Astronomical Journal 110 (1995) 880.

[6] P. C. Hewettand and V. Wild, Improved redshifts for SDSS quasar spectra, MNRAS 405 (2010) 2302. 
[7] M. F. Aller, P. A. Hughes, H. D. Aller, G. E. Latimer and T. Hovatta, Constraining the physical conditions in the jets of gamma-ray flaring blazars using centimeter-band polarimetry and radiative transfer simulations. $i$. data and models for 0420-014, OJ 287, and 1156+295, The Astrophysical Journal 791 (2014) 53.

[8] G. Ghisellini and F. Tavecchio, Fermi/LAT broad emmission line blazars, MNRAS 448 (2015) 1060.

[9] H. D. Aller, M. F. Aller, G. E. Latimer and P. E. Hodge, Spectra and linear polarizations of extragalactic variable sources at centimeter wavelenghs, The Astrophysical Journal Letters 59 (1985) 513.

[10] J.-Y. Wang, T. An, W. A. Baan and X.-L. Lu, Periodic radio variabilities of the blazar 1156+295: harmonic oscillations, Monthly Notices of the Royal Astronomical Society 443 (2014) 58.

[11] J. Aleksić, S. Ansoldi, L. A. Antonelli, P. Antoranz, A. Babic, P. Bangale et al., The major upgrade of the MAGIC telescopes, Part I: The hardware improvements and the commissioning of the system, Astroparticle Physics 72 (2016) 61.

[12] R. Zanin, E. Carmona, J. Sitarek, P. Colin, K. Frantzen, M. Gaug et al., MARS, the MAGIC analysis and reconstruction software, in Proc 33rd Int. Cosmic Ray Conference (ICRC2013): Rio de Janeiro, Brazil, p. 0773, 2013, http://inspirehep.net/record/1412925/files/icrc2013-0773.pdf.

[13] J. Aleksić, S. Ansoldi, L. A. Antonelli, P. Antoranz, A. Babic, P. Bangale et al., The major upgrade of the MAGIC telescopes, Part II: A performance study using observations of the Crab Nebula, Astroparticle Physics 72 (2016) 76 [1409.5594].

[14] A. Domínguez, J. R. Primack, D. J. Rosario, F. Prada, R. C. Gilmore, S. M. Faber et al., Extragalactic background light inferred from AEGIS galaxy-SED-type fractions, Monthly Notices of the Royal Astronomical Society 410 (2011) 2556.

[15] G. Ghisellini and F. Tavecchio, Canonical high-power blazars, MNRAS 397 (2009) 985. 